2019-1

\title{
Best Practices for Wake Model and Optimization Algorithm Selection in Wind Farm Layout Optimization
}

\author{
Nicholas F. Baker \\ Brigham Young University, nicholas.f.baker@gmail.com \\ Andrew P.J. Stanley \\ Brigham Young University, stanley_andrewpj@yahoo.com \\ Jared J. Thomas \\ Brigham Young University, jaredthomas68@gmail.com \\ Andrew Ning \\ Brigham Young University, aning@byu.edu \\ Katherine Dykes

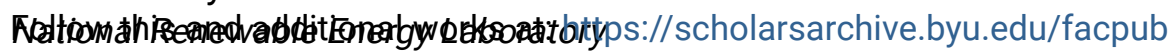 \\ Part of the Architectural Engineering Commons
}

\section{Original Publication Citation}

Baker, N. F., Stanley, A. P. J., Thomas, J. J., Ning, A., and Dykes, K., "Best Practices for Wake Model and Optimization Algorithm Selection in Wind Farm Layout Optimization," AIAA Scitech 2019 Forum, San Diego, CA, Jan. 2019. doi:10.2514/6.2019-0540

\section{BYU ScholarsArchive Citation}

Baker, Nicholas F.; Stanley, Andrew P.J.; Thomas, Jared J.; Ning, Andrew; and Dykes, Katherine, "Best Practices for Wake Model and Optimization Algorithm Selection in Wind Farm Layout Optimization" (2019). Faculty Publications. 2983.

https://scholarsarchive.byu.edu/facpub/2983

This Conference Paper is brought to you for free and open access by BYU ScholarsArchive. It has been accepted for inclusion in Faculty Publications by an authorized administrator of BYU ScholarsArchive. For more information, please contact ellen_amatangelo@byu.edu. 


\title{
Best Practices for Wake Model and Optimization Algorithm Selection in Wind Farm Layout Optimization
}

\author{
Nicholas F. Baker*, Andrew P. J. Stanley ${ }^{\dagger}$, Jared J. Thomas*, and Andrew Ning ${ }^{\ddagger}$ \\ Brigham Young University, Provo, Utah 84602. \\ Katherine Dykes ${ }^{\text {II }}$ \\ National Renewable Energy Laboratory, Golden, Colorado 80401
}

\begin{abstract}
This paper presents the results of two case studies regarding the wind farm layout optimization problem. We asked members of the computational optimization and wind communities to take part in the studies that we designed. Nine individuals participated. Case study 1 considered variations in optimization strategies for a given simple Gaussian wake model. Participants were provided with a wake model that outputs annual energy production (AEP) for an input set of wind turbine locations. Participants used an optimization method of their choosing to find an optimal wind farm layout. Case study 2 looked at trade-offs in performance resulting from variation in both physics model and optimization strategy. For case study 2, participants calculated AEP using a wake model of their choice while also using their chosen optimization method. Participants then used their wake model to calculate the AEP of all other participants' optimized layouts. Results for case study 1 show that the best optimal wind farm layouts in this study were achieved by participants who used gradient-based optimization methods. A front-runner emerged with the Sparse Nonlinear OPTimizer plus Wake Expansion Continuation (SNOPT+WEC) optimization method, which consistently discovered the highest submitted AEP. For case study 2, two participants found a similar layout that was judged to be superior by all five participants. It is unclear if the better solution resulted from an improved optimization process, or a wake model that was more amenable to optimization.
\end{abstract}

\section{Introduction}

$\mathrm{O}$ PTIMIZING turbine placement within a wind farm is a complex problem characterized by many local optima. The arge number of inter-dependent variables involved in wind farm layout optimization (WFLO) create a design space that is difficult to solve reliably. In this study, we designed and conducted a set of case studies to discover superior practices in solving the WFLO problem.

In WFLO, one of the most important factors is wake interaction between turbines [1]. Downstream rotors in the wake-affected area from upstream turbines experience reduced power production (due to the decreased wind velocity) and a shortened lifespan (due to increased turbulence intensity) [2]. Computational modeling can be used to optimize wind turbine layout and turbine attributes (such as hub height and rotor diameter) in order to reduce the effects of both the velocity deficit and the increased turbulence in the wake.

Two approaches have been taken to improve computational analysis in the WFLO problem. The first approach aims at improving the quality of individual models. The second approach is to improve the formulation of the optimization problem, as well as the algorithms used to perform the optimization [3].

To model the relevant fluid dynamics and wake interactions between turbines, researchers have taken two general approaches: (1) create computationally inexpensive simplified theoretical wake models, based on fundamental fluids principles or empirical data from the wind turbine wakes [2, 4, 5], or (2) use computationally expensive approaches, like the Reynolds-Averaged Navier-Stokes (RANS) equations, Large-Eddy Simulations (LES), or Direct Numerical Simulation (DNS), which increase the accuracy and granularity of their results [6].

\footnotetext{
*Masters Student, Department of Mechanical Engineering, 360 EB, Provo, UT 84602, AIAA Student Member

${ }^{\dagger}$ Ph.D. Candidate, Department of Mechanical Engineering, 360 EB, Provo, UT 84602, AIAA Student Member

${ }_{\ddagger}^{\ddagger}$ Ph.D. Student, Department of Mechanical Engineering, 360 EB, Provo, UT 84602, AIAA Student Member

$\S$ Assistant Professor, Department of Mechanical Engineering, 360 EB, Provo, UT 84602, AIAA Senior Member

"IS Senior Engineer, National Wind Technology Center, 15013 Denver West Pkwy, Golden, CO 80401
} 
Complex computational methods such as DNS or LES use the Navier-Stokes equations, which require extensive computational time that is prohibitive in multi-iterative testing. Simplified engineering wake models (EWMs) respond to this obstacle by making certain limiting physics assumptions that result in greatly reduced computational costs [1]. Optimization requires many evaluations, so reducing computational cost through simplifying methods like EWMs is necessary.

The second approach described by Padrón et al. regards optimization algorithms, which alter input variables in order to optimize output values. One can use such algorithms to optimize for many objectives in WFLO (i.e. turbine longevity, noise reduction, etc.), with a common objective being annual energy production (AEP). For a given EWM, the choice of optimization methods may be limited by characteristics of both the EWM and optimization method.

Optimization algorithms can be categorized as (1) gradient-based, or (2) gradient-free. Gradient-based algorithms require the governing functions to be continuous and differentiable in order to calculate derivatives. Flat models, those with large regions of zero-valued gradients, such as the Jensen "top-hat" [7] or FLORIS [8], may need a gradient-free algorithm for best performance. In contrast, Jensen's cosine model or Thomas' FLORIS improvement [9], both of which use differentiable functions, could use gradient-based optimizers [10]. Generally, gradient-based optimizers are fast and efficient at finding local optima for differentiable functions, but have difficulties when functions are noisy or if discontinuities are present [10]. Despite generally being slower, gradient-free methods can be used when gradients can't be obtained, or when obtaining the gradients is too costly [11]. Furthermore, within these gradient-based or gradient-free limitations, different optimization strategies have varying capacity to avoid local optima.

Sub-optimal turbine placement results in lost energy and potentially millions of forfeit dollars over the course of a wind farm's typical 20-year life-span [1]. Such errors could result from either an inaccurate wake model, or inefficient optimization algorithms. Mistakes in either of these two areas could be avoided with a clearer understanding of model and optimization best practices.

To better understand the effects of EWMs and optimization algorithms, we created two case studies. We solicited participant involvement from different research labs and private companies in industry currently working on both general optimization methods, as well as methods specific to solving the WFLO problem. The first case study isolated optimization techniques for a single simplified EWM; the second case study aimed to observe the differences when combining variations in the EWM and optimization method.

Though papers have been published that survey the state of the wind farm optimization (perhaps one of the most notable written by Herbert-Acero reviewing the current methodologies in the field [1]), our case studies are the first time an international collaboration has been conducted to comparatively and empirically analyze optimization methods and EWM selection on a representative WFLO problem.

Our case studies are created in support of the International Energy Agency's (IEA's) Wind Task 37 (IEA37). IEA37 coordinates international research activities centered around the analysis of wind power plants as holistic systems [12]. Our case studies concentrate on optimization at the farm-level, and so contribute to IEA37's integrated approach [12] to wind energy.

\section{Methodology}

To enable production of useful data, our case studies required a model wind farm with characteristics that were simultaneously restrictive enough to maintain simplicity, yet general enough to maintain relevance to more complex and realistic problems. The wind farm scenarios we selected to meet this criteria, and other details relevant to this project as a whole, are described below in Section II.A.

Many factors affect recommendations for superior turbine placement of a proposed wind farm. The two major factors we chose to study are 1) EWM characteristics and 2) optimization algorithm [1]. We designed two case studies in an attempt to quantify the effects of each of these choices.

For the first case study, in which the goal was to isolate variability in the optimization method, we pre-coded a representative wake model as a control variable and permitted participants to use any optimization strategy to alter turbine locations that would deliver the best annual energy production (AEP) for the farm. This is called case study 1 and is described below in Section II.B.

Isolating EWM variability proved more complicated. An EWM's compatibility with gradient-based or gradient-free optimization methods dictates which algorithms can be applied. As such, designing a case study that restricted participants to a single optimization algorithm would unnecessarily limit the scope of EWMs studied. For this reason, our second case study permitted not only participant selection of EWM but also the optimization algorithm. It is called case study 2 and is described below in Section II.C. 


\section{A. Common to Both Case Studies}

Though testing for different variables, certain wind farm attributes were common to both case studies. A brief list of these common variables is described below.

\section{Wind Turbine Specifications}

We used the IEA 3.35-MW reference turbine in all wind farms. The IEA 3.35-MW specifications are open source, and the turbine is designed as a baseline for onshore wind turbine specifications [13]. The power curve for the IEA 3.35-MW turbine is defined as shown in Eq. (1) and Fig. 1. The specifications of the turbine necessary for our simplified version of Bastankhah's Gaussian wake model (used in case study 1) are shown in Table 1.

$$
P(V)= \begin{cases}0 & V<V_{\text {cut-in }} \\ P_{\text {rated }}\left(\frac{V-V_{\text {cut-in }}}{V_{\text {rated }} V_{\text {cut-in }}}\right)^{3} & V_{\text {cut-in }} \leq V<V_{\text {rated }} \\ P_{\text {rated }} & V_{\text {rated }} \leq V<V_{\text {cut-out }} \\ 0 & V \geq V_{\text {cut-out }}\end{cases}
$$

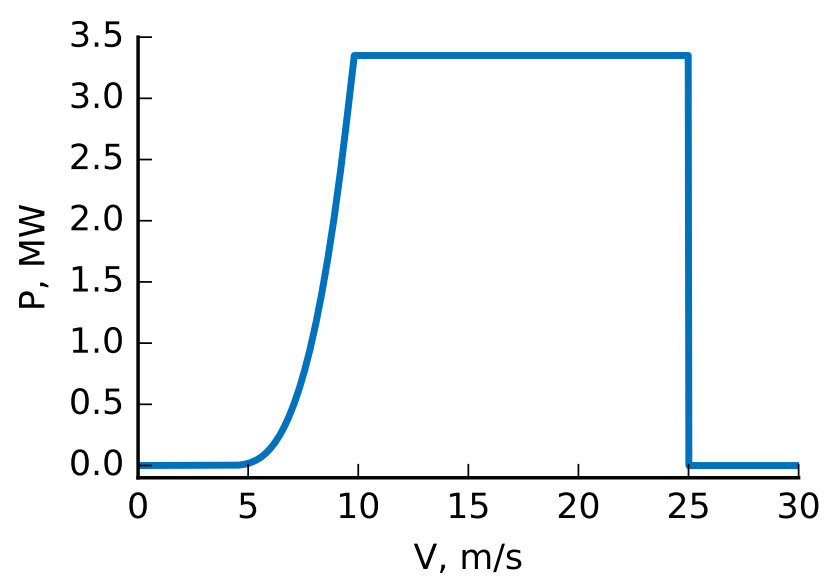

Figure 1 Graphical depiction of IEA's 3.35-MW onshore reference turbine's power curve.

Table 1 Attributes for IEA's 3.35-MW onshore reference turbine

\begin{tabular}{lrl}
\hline Rotor Diameter & 130 & $\mathrm{~m}$ \\
Turbine Rating & 3.35 & $\mathrm{MW}$ \\
Cut-In Wind Speed & 4 & $\mathrm{~m} / \mathrm{s}$ \\
Rated Wind Speed & 9.8 & $\mathrm{~m} / \mathrm{s}$ \\
Cut-Out Wind Speed & 25 & $\mathrm{~m} / \mathrm{s}$ \\
\hline
\end{tabular}

\section{Farm Geography}

To focus on optimization method and EWM variability, as well as to avoid introducing too many unnecessary variables, the wind farms for all scenarios were on flat and level terrain. To reduce boundary impacts on farm design, we chose a radially symmetric farm boundary. Turbine $(x, y)$ hub locations were restricted to be on or within the boundary radius. Turbines were further constrained to be no less than two rotor diameters apart from any other turbine. 
Farm diameter sizing for each scenario needed to be restrictive enough to avoid simply placing all turbines on the boundary but also permit meaningful turbine movement by the optimizers. Although the participants were not required to use the example starting layouts that we provided, we tried to provide reasonable example layouts by dispersing the turbines as much as possible in an orderly way. This was done by placing turbines in evenly spaced concentric rings. The boundary radii of the various wind farms we defined were selected to permit turbine placement in concentric rings with a minimum turbine spacing of five rotor diameters.

\section{Wind Attributes}

The wind distribution frequency and wind speed were the same for all wind farm scenarios in both case studies. Freestream wind velocity was constant in all wind directions, at $9.8 \mathrm{~m} / \mathrm{s}$, regardless of turbine location or time of day. This wind speed was used because it is the rated wind speed of the IEA 3.35-MW wind turbine. Using this incoming wind velocity maximized power production variability between wind turbines in the farm. In setting the scenario's freestream velocity for the turbine's rated wind speed, any wake effects moved air speeds down below rated power. With greater variability in the power production, more local optima would be experienced by participant optimizers. A lack of such local optima in a design space permits even ineffective optimizers to find a superior result. Since the presence of many local optima is a feature observed on many wind farm optimization problems, we strove to create such design spaces with our case study scenarios, as it allows us to test the exploration capabilities of the various optimization algorithms.

The selection of the wind rose was a major factor in the frequency and magnitude of local optima resulting from turbine placement. We selected a wind rose with an off-axis wind frequency distribution, binned for 16 directions. When we tested this wind rose against 1,000 randomized starting turbine locations, it gave few optimized results with relatively high AEP values. We interpreted this to be indicative of the presence of many local optima. The wind rose we used is depicted in Fig. 2, in polar coordinates. In this figure, a greater magnitude in the radial direction from the origin indicates a higher wind frequency from that specific direction.

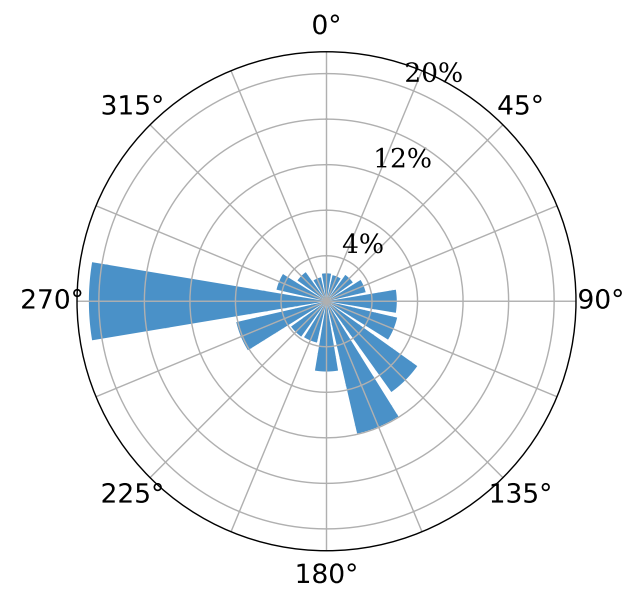

Figure 2 The wind frequency distribution for our case studies.

\section{B. Case Study 1: Optimization Only}

The purpose of this case study was to determine the best optimization practices for WFLO, using a single representative EWM. We selected a generalized wake model that both gradient-based and gradient-free optimization algorithms could use and that was computationally inexpensive in comparison to LES and DNS methods. 


\section{Wake Model}

The wake model selected for case study 1 was a simplified version of Bastankhah's Gaussian wake model [14, 15]. This wake model is described Eq. (2).

$$
\frac{\Delta U}{U_{\infty}}=\left(1-\sqrt{1-\frac{C_{T}}{8 \sigma_{y}^{2} / D^{2}}}\right) \exp \left(-0.5\left(\frac{y-\delta}{\sigma_{y}}\right)^{2}\right)
$$

In Eq. (2), $\Delta U / U_{\infty}$ is the wake velocity deficit, $C_{T}=8 / 9$ is the thrust coefficient, $y-\delta$ is the distance of the point of interest from the wake center in the cross-stream horizontal direction, $D$ is the turbine diameter, and $\sigma_{y}$ is the standard deviation of the wake spread in the cross-stream horizontal direction as defined in Eq. (3):

$$
\sigma_{y}=\left(k_{y} x\right)+\frac{D}{\sqrt{8}}
$$

In Eq. (3), $x$ is the downstream distance from the turbine generating the wake to the turbine of interest, and $D$ is the turbine diameter. The variable $k_{y}$ is determined as a function of turbulence intensity $(I)$. In this case study turbulence intensity was treated as a constant of 0.075 , and we therefore used a corresponding $k_{y}$ of $0.0324555[15,16]$.

Increasing turbulence intensity has numerous effects and draws attention away from the main purpose of this case study, which was to observe the differences of optimization strategies. For the wake model we used (shown in Eq. (2)), increasing the turbulence intensity widened the wake cone, but second and third order effects are unknown. As such, this first IEA37 set of case studies used a very low intensity in an attempt to minimize the considered variables.

\section{Farm Sizes}

Variability in wind farm size (and thus number of design variables) affects optimization algorithm performance. To study how increased farm size (i.e. design space complexity) impacts the performance of optimization algorithms, three wind farm sizes were specified in case study 1 . The three wind farms had 16, 36, and 64 turbines, respectively. The three farm boundary radii were $1300 \mathrm{~m}, 2000 \mathrm{~m}$, and $3000 \mathrm{~m}$, respectively. The boundary radii were determined in the manner described previously in Section II.A.2. The turbine numbers were selected as perfect squares that roughly double in size. Perfect squares were used to permit participants to use even grid turbine arrangements, if desired.

\section{Supplied Code}

We provided participants with a link to a GitHub repository* which included files with the following contents:

- Turbine characteristics, wind frequency, and wind speed in IEA 37's . yaml schema

- Example turbine layouts for each farm size (in . yaml format)

- Python parsers of the .yaml schema

- Python target function to calculate AEP (given . yaml turbine locations and farm attributes)

We selected the programming language Python, since it is widely used by researchers in the industry, and is open source. Participants were allowed to alter our specific code implementation or replicate the provided model in another language to speed up the code or for compatibility with their optimization methods. This was with the understanding, however, that final wind farm layouts would be evaluated with the original Python code that we provided.

\section{Case Study 2: Combined Physics Model/Optimization Algorithm}

The intent of this case study was to assess both the effects that different optimization methods and physics model approximations have on turbine location recommendations. Case study 2 differs from case study 1 in that 1 ) no wake model was provided and 2) only a single wind farm size was to be optimized. Participants were free to choose their preferred EWM and optimization method combination.

\section{Wake Model}

Unlike case study 1, participant-reported AEP values were not comparable, since each participant used a different EWMs to calculate AEP. To help us make fair comparisons and conclusions, we conducted a cross-comparison of results between participants. For the cross-comparison, each participant provided their optimal turbine layout in the

\footnotetext{
*https://github.com/byuflowlab/iea37-wflo-casestudies
} 
standardized .yaml format. Each participant was then provided with every other participants' optimized layout file. Participants then used their own wake model to calculate the AEP of the other participant's proposed farm layouts with their EWMs. From this portion of the case study, we hoped to learn if any participants' results were consistently seen as superior by other EWMs.

\section{Farm Attributes}

The wind farm size for the combined case study was limited to nine turbines. We did this to limit the computation time requirements when assessing results in a standardized LES, discussed later in Section IV. We used the previously described method under Section II.A. 2 to determine the boundary radius, which for the 9 turbine case is $900 \mathrm{~m}$. The wind rose and wind speed were the same as in case study 1.

\section{Results}

There were 10 submissions for case study 1. One participant submitted twice, using a different optimization method for each submission. For anonymity, each submission was assigned a number. We refer to each submission below by this submission number (i.e., sub1, ..., sub10, etc.). For case study 2, there were 5 participant submissions. All five participants for case study 2 also submitted for case study 1, though were not required to do so. For ease of comparison, we assigned their submissions the same numbers from that case study as well (i.e., subl - sub5 are from the same individual participants for both case study 1 and case study 2).

It should be noted that four of the authors submitted results for case study 1 as participants, and three submitted for case study 2. The primary author developed and collected data on the case study, but did so without sharing results with other participants including the other authors.

With submissions, was asked participants to also report their hardware specifications, and performance data including function calls, wall time, and number of optimizations run. Some of the questions in the exit questionnaire were not worded clearly enough, leading to different interpretations of time and function call reporting. On case showing wall time is shown below.

\section{A. Case Study 1: Optimization Only}

Participants ran the optimization algorithm of their choosing using our supplied AEP function or a functional equivalent in another language. The AEP results and rankings are given below in Tables 2 to 4 .

\section{Data}

Tables 2 to 4 display the final AEP data of all participant-proposed optimal turbine layouts. The Python module we supplied, which uses the simplified Bastankhah wake model, was used for all AEP calculations. Submissions were ranked from highest to lowest resultant AEP values. Also listed in the tables are the submission number (sub\#), whether a gradient-based $(G)$ or gradient-free $(G F)$ optimization method was used, and the relative percentage increase of AEP (Increase) from the provided example layout's AEP.

\section{General Trends}

As a general trend, gradient-based methods performed better in discovering a relative optima, especially for smaller farm sizes. Some gradient-based algorithms improved in comparative AEP ranking as the number of design variables increased (sub10, sub3), while others degraded (sub5, sub8). Simultaneously, one gradient-free algorithm increaseed in effectiveness as design variables increased (sub3), while others competed for lowest comparative performance, regardless of farm size (sub6, sub7, sub9).

Despite these multivariate results, one clear front-runner did emerge. Regardless of wind farm size, sub4's algorithm consistently discovered turbine placements that delivered an AEP superior to all other participants. A summary of sub4's method is included in a following section.

Also of note, as the number of design variables increased, the relative disparity between proposed optimal AEPs likewise diverged. For the 16-turbine case, the highest result was $7.88 \%$ better than the lowest. For the 36 and 64 cases, the highest result was $11.45 \%$ and $13.54 \%$ better than the lowest, respectively. 
Table 216 turbine scenario participant results

\begin{tabular}{rlccrr}
\hline Rank & Algorithm & sub\# & Grad. & AEP & Increase \\
\hline 1 & SNOPT+WEC & 4 & G & 418924.4064 & $14.17 \%$ \\
2 & fmincon & 5 & G & 414141.2938 & $12.86 \%$ \\
3 & SNOPT & 8 & G & 412251.1945 & $12.35 \%$ \\
4 & SNOPT & 1 & G & 411182.2200 & $12.06 \%$ \\
5 & Preconditioned Sequential Quadratic Programming & 2 & G & 409689.4417 & $11.65 \%$ \\
6 & Multistart Interior-Point & 10 & G & 408360.7813 & $11.29 \%$ \\
7 & Full Pseudo-Gradient Approach & 3 & GF & 402318.7567 & $9.64 \%$ \\
8 & Basic Genetic Algorithm & 7 & GF & 392587.8580 & $6.99 \%$ \\
9 & Simple Particle Swarm Optimization & 6 & GF & 388758.3573 & $5.95 \%$ \\
10 & Simple Pseudo-Gradient Approach & 9 & GF & 388342.7004 & $5.83 \%$ \\
11 & (Example Layout) & - & - & 366941.5712 & - \\
\hline
\end{tabular}

Table 336 turbine scenario participant results

\begin{tabular}{rlcccr}
\hline Rank & Algorithm & sub\# & Grad. & AEP & Increase \\
\hline 1 & SNOPT+WEC & 4 & G & 863676.2993 & $17.05 \%$ \\
2 & Multistart Interior-Point & 10 & G & 851631.9310 & $15.42 \%$ \\
3 & Preconditioned Sequential Quadratic Programming & 2 & G & 849369.7863 & $15.11 \%$ \\
4 & SNOPT & 8 & G & 846357.8142 & $14.70 \%$ \\
5 & SNOPT & 1 & G & 844281.1609 & $14.42 \%$ \\
6 & Full Pseudo-Gradient Approach & 3 & GF & 828745.5992 & $12.31 \%$ \\
7 & fmincon & 5 & G & 820394.2402 & $11.18 \%$ \\
8 & Simple Pseudo-Gradient Approach & 9 & GF & 813544.2105 & $10.25 \%$ \\
9 & Basic Genetic Algorithm & 7 & GF & 777475.7827 & $5.37 \%$ \\
10 & Simple Particle Swarm Optimization & 6 & GF & 776000.1425 & $5.17 \%$ \\
11 & (Example Layout) & - & - & 737883.0985 & \\
\hline
\end{tabular}

Table 464 turbine scenario participant results

\begin{tabular}{rlcccr}
\hline Rank & Algorithm & sub\# & Grad. & AEP & Increase \\
\hline 1 & SNOPT+WEC & 4 & G & 1513311.1936 & $16.86 \%$ \\
2 & Preconditioned Sequential Quadratic Programming & 2 & $\mathrm{G}$ & 1506388.4151 & $16.36 \%$ \\
3 & Multistart Interior-Point & 10 & $\mathrm{G}$ & 1480850.9759 & $14.35 \%$ \\
4 & SNOPT & 1 & $\mathrm{G}$ & 1476689.6627 & $14.03 \%$ \\
5 & Full Pseudo-Gradient Approach & 3 & $\mathrm{GF}$ & 1455075.6084 & $12.36 \%$ \\
6 & SNOPT & 8 & $\mathrm{G}$ & 1445967.3772 & $11.66 \%$ \\
7 & Simple Pseudo-Gradient Approach & 9 & $\mathrm{GF}$ & 1422268.7144 & $9.82 \%$ \\
8 & Simple Particle Swarm Optimization & 6 & $\mathrm{GF}$ & 1364943.0077 & $5.40 \%$ \\
9 & fmincon & 5 & $\mathrm{G}$ & 1336164.5498 & $3.18 \%$ \\
10 & Basic Genetic Algorithm & 7 & $\mathrm{GF}$ & 1332883.4328 & $2.93 \%$ \\
11 & (Example Layout) & - & - & 1294974.2977 & - \\
\hline
\end{tabular}




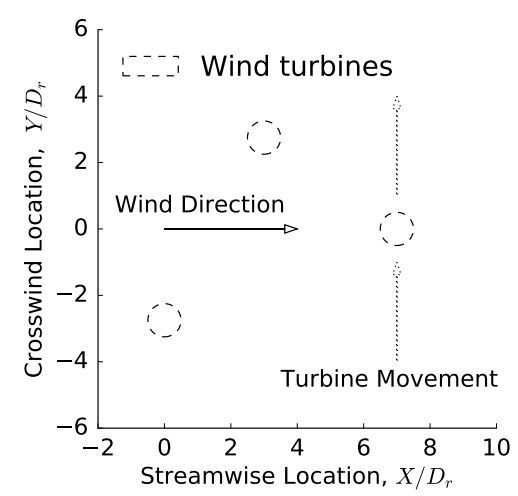

Figure 3 Simple design space used to demonstrate the effects of the relaxation factor, $\xi$, on the wind farm layout design space. [15]

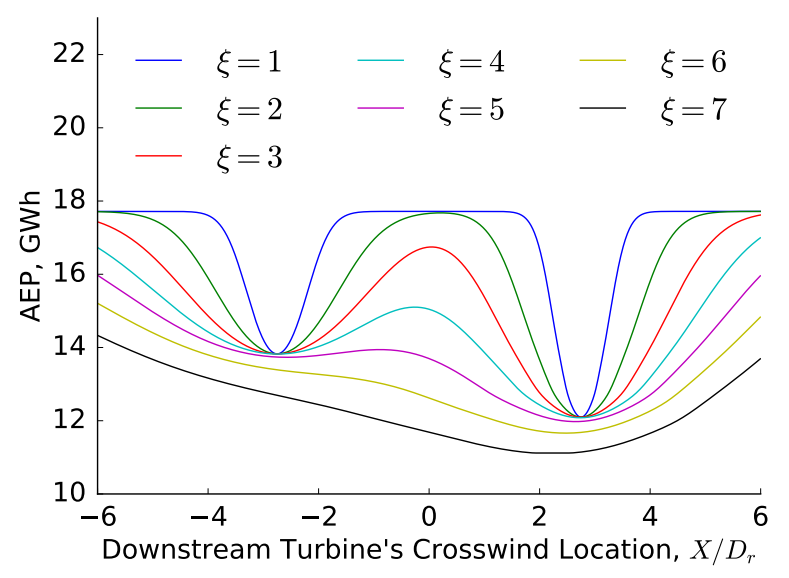

Figure 4 The impact of the wake relaxation factor, $\xi$. One turbine was moved across the wakes of two upstream turbines (see Fig. 3). [15]

\section{Analysis of Best Results}

For all three farm sizes, the superior method was implemented by sub4, using a gradient-based method. Coded in Python and FORTRAN, it combined SNOPT [17] with a method called Wake Expansion Continuation (WEC) [15]. Running 200 optimizations, sub4 had one optimization run start from the provided example layout, and the other 199 use randomized turbine starting locations within the farm boundary.

The WEC method is specifically designed to reduce the multimodality found in wind farm layout optimization. In the cited paper [15], it is shown to be a method which converts design spaces with many local minima into curves approaching convexity, allowing gradient-based optimizations to more easily find the better solutions. An example of such "relaxation" to convexity is included in Figs. 3 and 4, reproduced with permission.

The effect of the WEC method on a simple design space is shown in Figs. 3 and 4. WEC smooths out the local optima, making better solutions available to gradient-based optimization methods. As the authors Thomas and Ning state, "Larger values of $\xi$ allow the smaller local optima to disappear completely. Smaller values of $\xi$ allow for more accurate wake widths but with an increase in the number and magnitude of local optima." [15]. We suspect that the WEC method for reducing the multimodality of the design space is why sub4's optimizations found superior layouts as compared with the other methods used.

\section{Discussion}

Though sub4 consistently found the superior AEP relative to the other participants, sub2's results demonstrated a trend closing the gap as the number of design variables increased. For the 16 turbine case, sub4 was $2.5 \%$ better than sub2's results. For the 36 and 64 cases, sub4 was $1.68 \%$ and $0.46 \%$ better, respectively. It should be noted, however, that at the current average U.S. rate [18] of roughly $\$ 0.13$ for a $\mathrm{kWh}$ (or $\$ 133$ per $\mathrm{MWh}$ ), the income difference between the AEPs of sub4 and sub2 in the 64 turbine case, though only $0.46 \%$, equates to a difference of a little under $\$ 1$ million per year. Since sub2's Preconditioned Sequential Programming (PSQP) method steadily closed the gap, a future study should test even larger wind farm sizes. This could determine if the PSQP algorithm could eventually outperform the SNOPT+WEC method when a certain number of design variables are reached, or if there is an upper limit or convergence to this trend.

Though the majority of participants used random starts for each optimizaiton, sub2's method of "warm starting" performed progressively well, especially as the number of design variables increased. Taking a starting set of turbine coordinates, $s u b 2$ rotated the layout in $\pi / 6$ steps. These rotations created the starting geometry for subsequent iterations. Though not precisely "intuitive" starts, they are more intelligently designed than pure randomized locations. As discussed above, sub2 did perform increasingly well compared to other methods (ranking 2nd for the 64-turbine case).

Translating the provided AEP target function proved helpful in speeding up the computations and allowing for greater exploration. At least two participants translated the target Python file into FORTRAN, one into Julia, and one 
altered it within Python by converting loops into vectorized statements. In testing, these reimplementations sped up the analysis time by at least an order of magnitude.

In our survey we asked participants to self-report time and iteration count for their optimizations. However, the question was not clearly worded resulting in different interpretations. Some reported the time for their best optimization run, while others included total time include exploratory runs, multistarts, or other iterative approaches (the latter was intended). Also, because we did not warn users that this information would be requested in the survey, some of the numbers were not recorded during optimization and were simply estimated. As an example, self-reported optimization time for the 64 turbine case is shown in Fig. 5 labeled by submission number. Given the limitations in reporting described above, no real conclusions can be drawn at this time, but the data is provided to give a general sense of the algorithmic times.

\section{B. Case Study 2: Combined Physics Model/Optimization Algorithm}

For case study 2, participants ran both the optimization algorithm and wake model of their choosing. There was no restrictions on programming language for either the wake model or optimization algorithm, but results of optimal turbine layouts were to be submitted in the .yaml format supplied in the case study 1 examples.

Because participants used different wake models, AEP values reported cannot be fairly compared between participants. Results were therefore judged on cross-comparison calculations.

\section{Data}

The cross-comparison displays some interesting trends. Tables 5 to 9 show how each submission's wake models ranked the proposed optimal turbine layouts for the other 4 submissions. Each submission's ranking of its own layout is in bold. The penultimate column in each table is the submission number of the layout being cross-compared (cc-sub\#). So submission 4's analysis of submission 2's layout would be found in sub4's table, with 2 in the cc-sub\# column. The last column is the percentage difference (Difference) from the reporting submission's submitted layout. A positive value here indicates a better AEP, a negative value indicates a worse one.

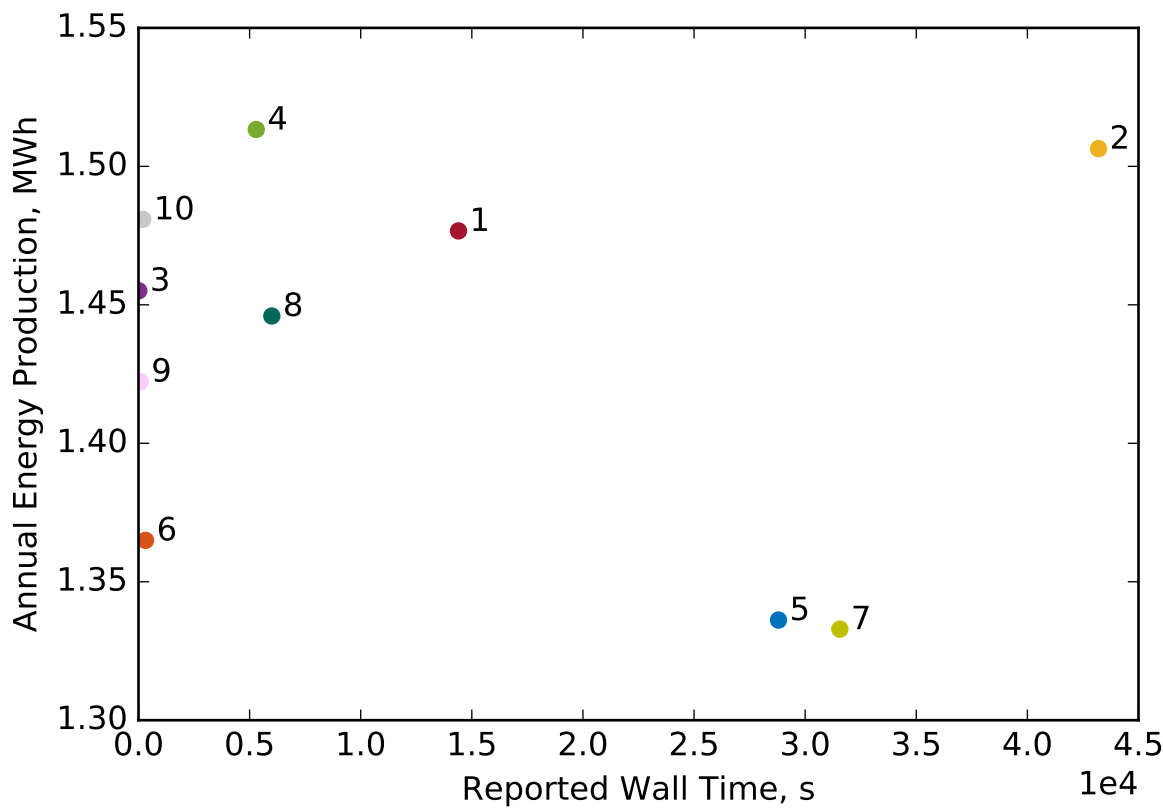

Figure 5 AEP vs wall time, 64 turbine scenario. Submission numbers placed next to reported values. 
Table 5 Cross-comparison results of sub1

\begin{tabular}{rllllr}
\hline Rank & Wake Model & Algorithm & AEP & cc-sub\# & Difference \\
\hline 1 & Bastankhah & SNOPT+WEC & 262350.319 & 4 & $0.624 \%$ \\
2 & Simplified Bastankhah & fmincon & 262282.416 & 5 & $0.598 \%$ \\
3 & FLORISSE 3D & SNOPT & $\mathbf{2 6 0 7 2 2 . 2 9 5}$ & $\mathbf{1}$ & - \\
4 & Bastankhah & Full Pseudo-Gradient Approach & 260640.906 & 3 & $-0.031 \%$ \\
5 & Park2 & PSQP & 248215.024 & 2 & $-4.797 \%$ \\
\hline
\end{tabular}

Table 6 Cross-comparison results of sub2

\begin{tabular}{rllllr}
\hline Rank & Wake Model & Algorithm & AEP & cc-sub\# & Difference \\
\hline 1 & Bastankhah & SNOPT+WEC & 250464.9732 & 4 & $5.975 \%$ \\
2 & Simplified Bastankhah & fmincon & 250249.0259 & 5 & $5.884 \%$ \\
3 & Bastankhah & Full Pseudo-Gradient Approach & 247812.0522 & 3 & $4.853 \%$ \\
4 & FLORISSE 3D & SNOPT & 240309.5850 & 1 & $1.678 \%$ \\
5 & Park2 & PSQP & $\mathbf{2 3 6 3 4 2 . 7 9 9}$ & $\mathbf{2}$ & - \\
\hline
\end{tabular}

Table 7 Cross-comparison results of sub3

\begin{tabular}{rllllr}
\hline Rank & Wake Model & Algorithm & AEP & cc-sub\# & Difference \\
\hline 1 & Simplified Bastankhah & fmincon & 247109.5234 & 5 & $0.590 \%$ \\
2 & Bastankhah & SNOPT+WEC & 246942.3767 & 4 & $0.522 \%$ \\
3 & Bastankhah & Full Pseudo-Gradient Approach & $\mathbf{2 4 5 6 5 9 . 4 1 2 4}$ & $\mathbf{3}$ & - \\
4 & Park2 & PSQP & 242431.5431 & 2 & $-1.314 \%$ \\
5 & FLORISSE 3D & SNOPT & 237548.6622 & 1 & $-3.302 \%$ \\
\hline
\end{tabular}

Table 8 Cross-comparison results of $s u b 4$

\begin{tabular}{rllllr}
\hline Rank & Wake Model & Algorithm & AEP & cc-sub\# & Difference \\
\hline 1 & Bastankhah & SNOPT+WEC & $\mathbf{2 5 7 7 9 0 . 1 9 2 4}$ & $\mathbf{4}$ & - \\
2 & Simplified Bastankhah & fmincon & 257663.4068 & 5 & $-0.049 \%$ \\
3 & Bastankhah & Full Pseudo-Gradient Approach & 255063.8201 & 3 & $-1.058 \%$ \\
4 & FLORISSE 3D & SNOPT & 251776.7157 & 1 & $-2.333 \%$ \\
5 & Park2 & PSQP & 239612.8223 & 2 & $-7.051 \%$ \\
\hline
\end{tabular}

Table 9 Cross-comparison results of sub5

\begin{tabular}{rllllr}
\hline Rank & Wake Model & Algorithm & AEP & cc-sub\# & Difference \\
\hline 1 & Simplified Bastankhah & fmincon & $\mathbf{2 5 1 7 7 1 . 9 0 6 7}$ & $\mathbf{5}$ & - \\
2 & Bastankhah & SNOPT+WEC & 251697.7126 & 4 & $-0.029 \%$ \\
3 & Bastankhah & Full Pseudo-Gradient Approach & 249829.2199 & 3 & $-0.772 \%$ \\
4 & FLORISSE 3D & SNOPT & 246503.8323 & 1 & $-2.092 \%$ \\
5 & Park2 & PSQP & 239482.6767 & 2 & $-4.881 \%$ \\
\hline
\end{tabular}




\section{General Trends}

We expected participants to rank their own layout as superior to the others. Each wake model accounts for different fluids phenomena, and what one wake model considers an optimal layout, another may not. An example of this is if one EWM predicts a wake deficit due to some factor such as vorticity or turbulence. A turbine placed downstream under such a model would, under a more simplistic wake model not accounting for this phenomena (such as the Jensen's model [7]), would feel the full brunt of the wake and deliver a suboptimal AEP.

Unexpectedly, only sub4 and sub5 found their own layouts to be superior to the other participants. Furthermore, all other participants also found sub4 and sub5's layouts superior to their own, though to varying degrees. Three participants (including sub4) found sub4 to have the highest AEP-producing layout. The other two participants found sub5 to have the highest AEP-producing layout.

\section{Analysis of Best Results}

Within expectations, sub4 and sub5 ranked their own layouts superior to all other participant results. Two correlations are important to note regarding sub4 and sub5. First, both used variations of the same wake model. From case study 1 , sub5 used the simplified Gaussian wake model previously described [14, 15]. Though sub4 also used the Gaussian wake model [14], sub4 combined it with the model created by Niayifar and Porté-Agel [16], supplemented by the WEC method described earlier. Furthermore, sub4 also accounted for wind shear and local turbulence intensity. Neither of these factors were accounted for by sub5. The second factor to note is that despite using very similar wake models, sub4 and $s u b 5$ used different gradient-based optimization algorithms that reached very similar conclusions.

As can be seen in the figures included in the Appendix, sub4 and sub5 found nearly identical optimal turbine placements. Though appearing identical, the actual coordinates do indeed differ, enough so to result in different AEP calculations shown in the tables above.

Without LES data, the conclusions able to be drawn from the cross-comparison analysis are limited. Reasons that both sub4 and sub5 were found by the other participant wake models to have superior placement could be a result of more efficient optimization methods, better coupling between optimization method and wake model, or wake model superiority. The reason that these minima existed within the other wake models (resulting in a higher computed AEP by those models), yet were nevertheless undiscovered in their optimizations, is inconclusive in telling us which it is.

Both sub4 and sub5 used similar wake models but very different optimization methods. Coding in MATLAB, sub5 did 1,000 random starts and used MATLAB's fmincon (which uses a finite difference method to find gradients) to optimize for a minimum. Using a combination of Python and FORTRAN, sub4 ran 1 optimization with a user-selected initial turbine configuration, and randomized the turbine starting locations for another 199 to make 200 optimizations altogether. SNOPT's SQP algorithm (using algorithmic differentiation to obtain gradients) was sub4's implemented optimizer.

Of note, from trends seen above in case study 1, sub5's optimization methods demonstrated superior performance for small design variable sizes but comparatively degraded as the wind farm size increases. The superior performance of this wake model and optimization method combination for this small farm may not be representative of performance on larger wind farms.

\section{Discussion}

Participants of earlier case studies were critical of wind farm scenarios where non-novel, simplistic layouts (such as all turbines on the boundary border) are optimal. The small farm radius with few turbines given for this case study seems to have fallen into this category. Even with our very simple case studies, the participants found very different results.

Many factors could have led to these shortfalls (i.e., inferior optimization methods, lack of sufficient iterations, lack of sufficient wall time, etc.), and further testing would need to be done to discover which factors majorly contributed to the outcome.

\section{Conclusion}

We created two case studies to better understand the effects of EWMs and optimization algorithms as applied to the WFLO problem. Case study 1 focused on optimization methods, and received 10 submissions. Case study 2 studied the combination of EWM and optimization method, and received 5 participant submissions.

Results from case study 1 show that sub4s use of SNOPT+WEC delivered superior results for the tested wind farms with 16, 36, and 64 turbines. Although information on this method continues to be produced, the initial paper written by 
Thomas and Ning [15] describes this method. Regarding sub2's PSQP method, though it showed a trend of increased performance that may surpass SNOPT+WEC for wind farms of sizes larger than 64 , further testing is required to validate this pattern.

Case study 2 demonstrated that, for wind farms of small area with few turbines, placement on the wind farm boundary delivers superior AEP. Three of the five participants reported in their cross-comparison that others found superior optima to theirs, indicating that their optimization methods became trapped in a local optima. It is unclear if the difference was caused by a difference in the optimization approach, or in the wake models' suitability for optimization. Further investigation is needed in comparing the approaches in more detail. One approach we would like to pursue is to run all participant-reported optimized turbine locations through a higher-fidelity simulation, like a Large Eddy Simulation.

Though we are happy with the level of participation in the case studies, a larger participant sample size with different methods may provide more informative data or display other novel and superior methods. To refine our data collection process, we plan on running another round of results for these case studies in the near future. In future case studies, more precise wording is needed for questions regarding participant data, especially pertaining to wall time and function calls. Also, before beginning the case studies, participants should know exactly what information to track and save. 


\section{Acknowledgments}

This work was authored [in part] by the National Renewable Energy Laboratory, operated by Alliance for Sustainable Energy, LLC, for the U.S. Department of Energy (DOE) under Contract No. DE-AC36-08GO28308. Funding provided by the U.S. Department of Energy Office of Energy Efficiency and Renewable Energy Wind Energy Technologies Office. The views expressed in the article do not necessarily represent the views of the DOE or the U.S. Government. The U.S. Government retains and the publisher, by accepting the article for publication, acknowledges that the U.S. Government retains a nonexclusive, paid-up, irrevocable, worldwide license to publish or reproduce the published form of this work, or allow others to do so, for U.S. Government purposes.

The authors gratefully acknowledge the following individuals, in alphabetical order, as submitting participants in the case study:

- Tim Camp, Director, Turbine Engineering

- Abhinav Prakash, Ph.D. Student, Texas A\&M University

- Erik Quaeghebeur, Dr.ir, Delft University of Technology

- Sebastian, Sanchez Perez Moreno, Ph.D. Student, Delft University of Technology

- Landon Wiley, M.Sc Student, Brigham Young University 


\section{Appendix}

This appendix contains plots of the optimized wind farm layouts submitted by all participants for both case studies. The optimized layouts for case study 1 are shown in Figs. 6 to 8 for the 16, 36, and 64 turbine wind farms, respectively. The optimized layouts for case study 2 are shown in Fig. 9.

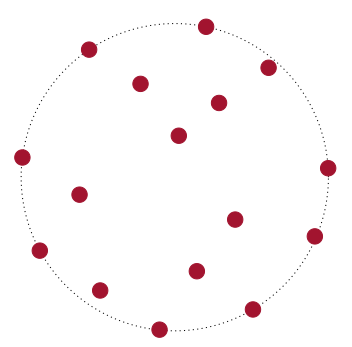

(a) subl

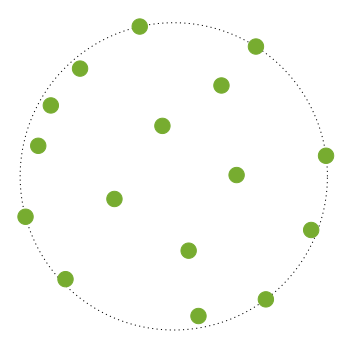

(d) $s u b 4$

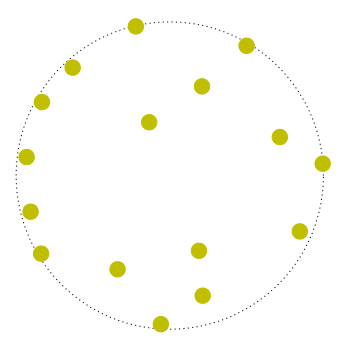

(g) $s u b 7$

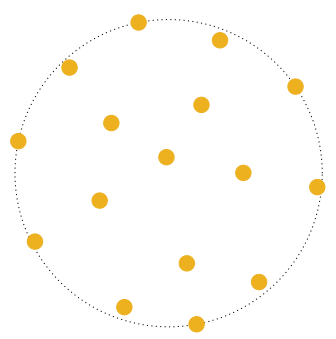

(b) $s u b 2$

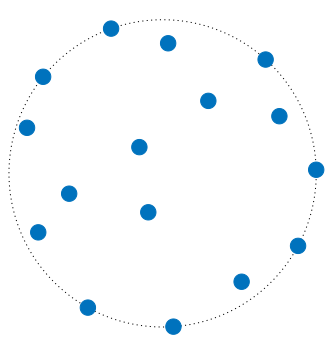

(e) sub5

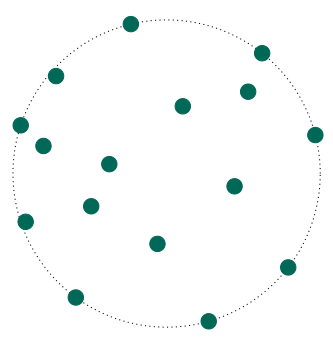

(h) $\operatorname{sub8}$

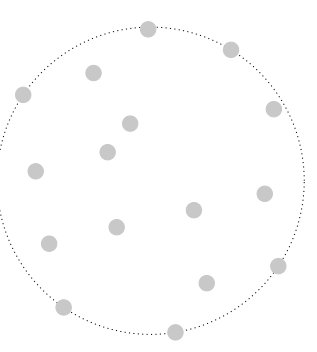

(j) $\operatorname{sub10}$

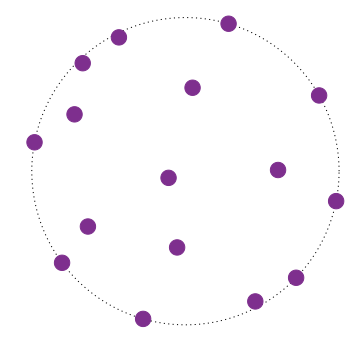

(c) $s u b 3$

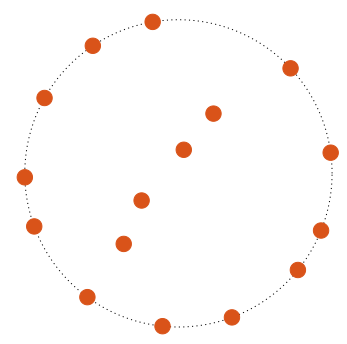

(f) $s u b 6$

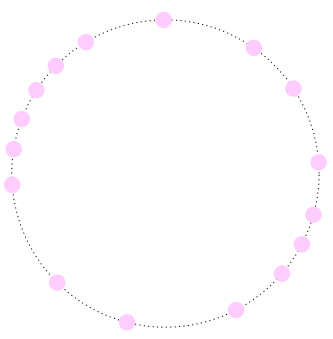

(i) $\operatorname{sub} 9$

Figure 6 Case study 1: optimized wind farm layouts with 16 wind turbines. 


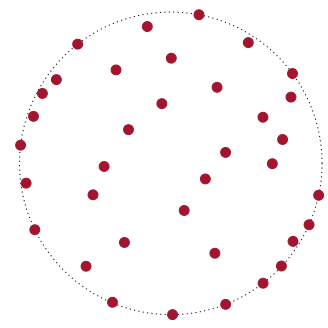

(a) $s u b 1$

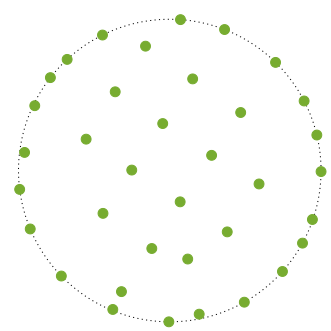

(d) sub4

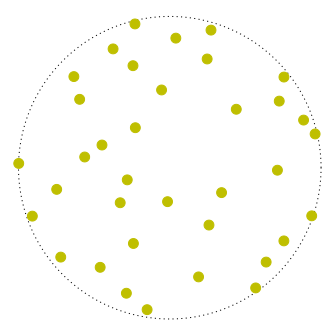

(g) $s u b 7$

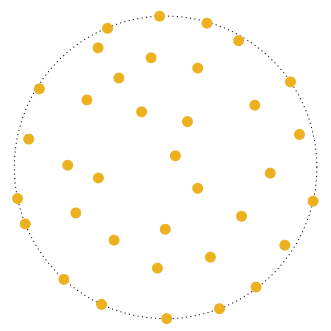

(b) sub2

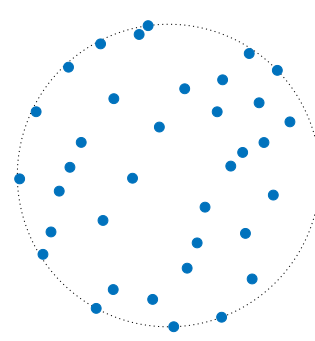

(e) $s u b 5$

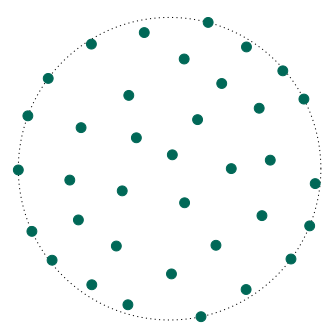

(h) $s u b 8$

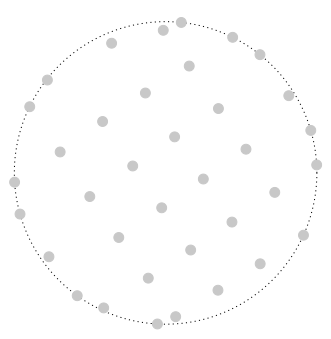

(j) $s u b 10$

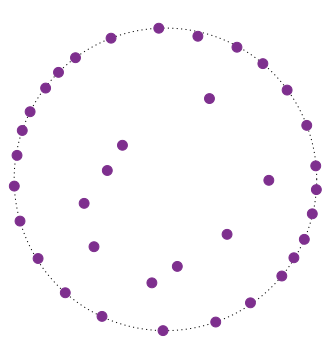

(c) $s u b 3$

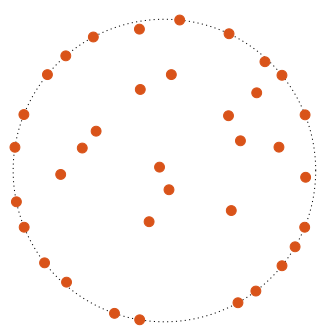

(f) $s u b 6$

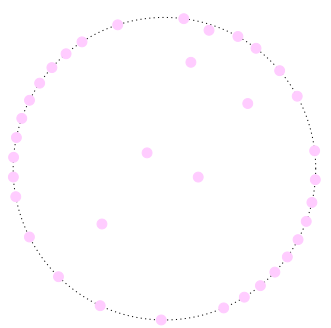

(i) $s u b 9$

Figure 7 Case study 1: optimized wind farm layouts with 36 wind turbines. 


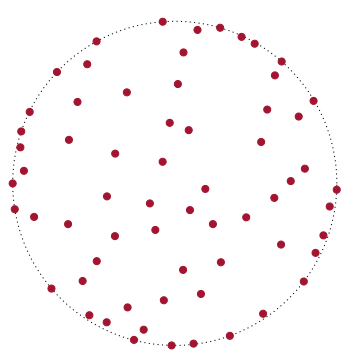

(a) $s u b 1$

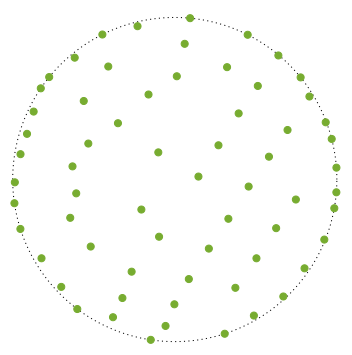

(d) $s u b 4$

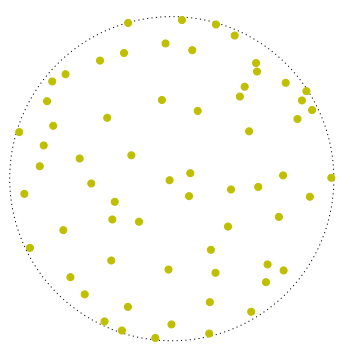

(g) $s u b 7$

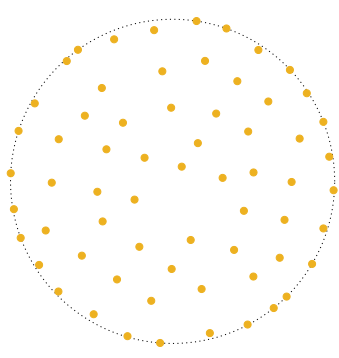

(b) $s u b 2$

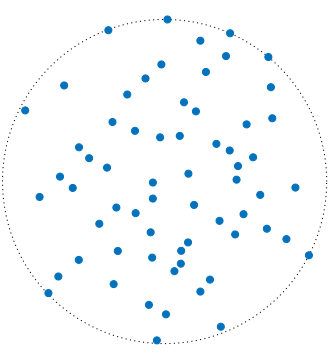

(e) $s u b 5$

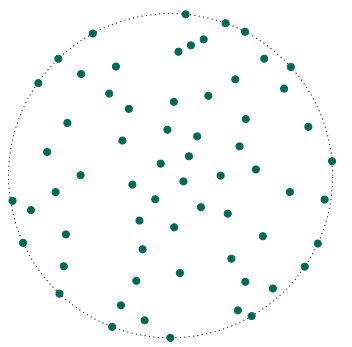

(h) $s u b 8$

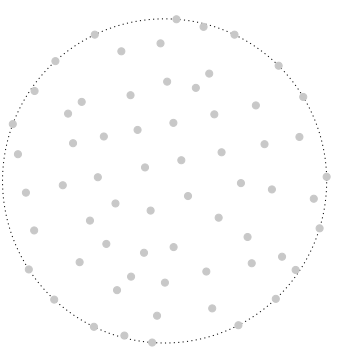

(j) $s u b 10$

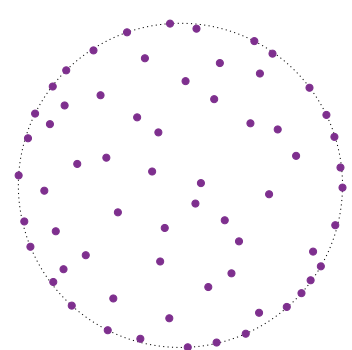

(c) $s u b 3$

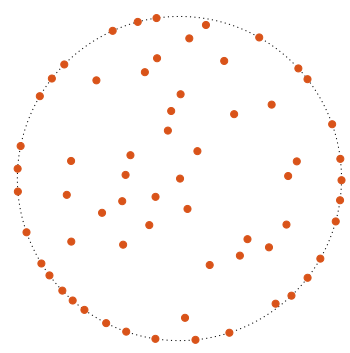

(f) $s u b 6$

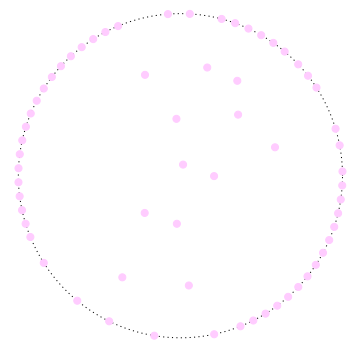

(i) $s u b 9$

Figure 8 Case study 1: optimized wind farm layouts with 64 wind turbines. 


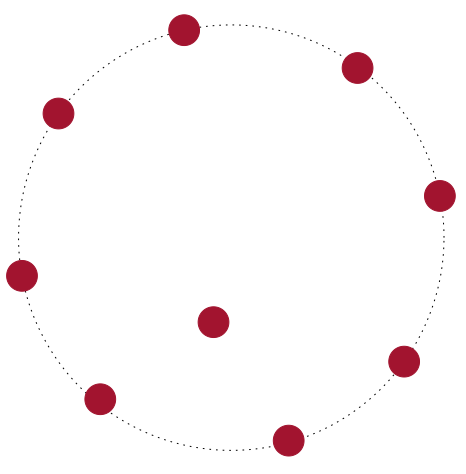

(a) subl

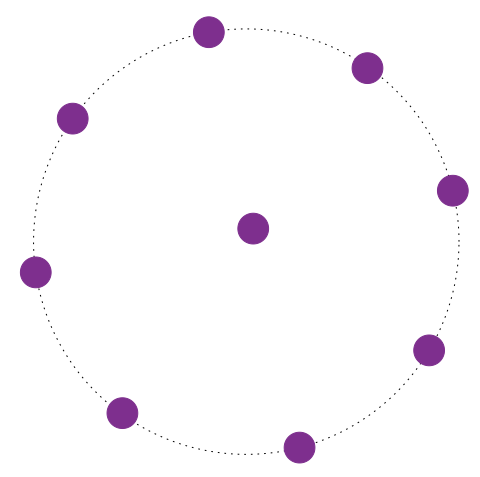

(c) $\operatorname{sub3}$

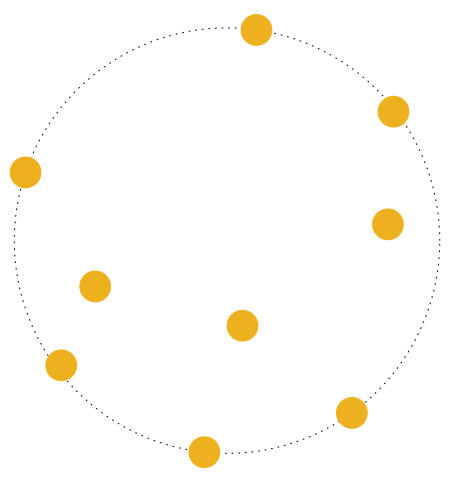

(b) $s u b 2$

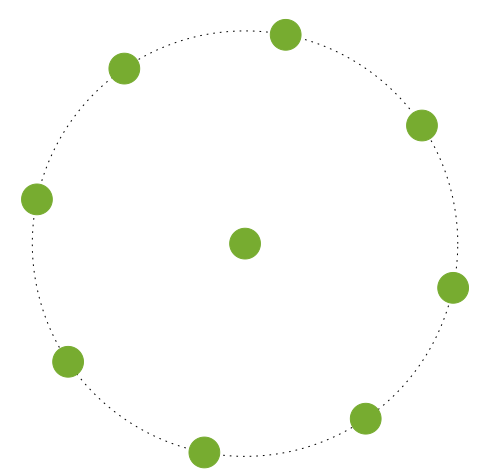

(d) $s u b 4$

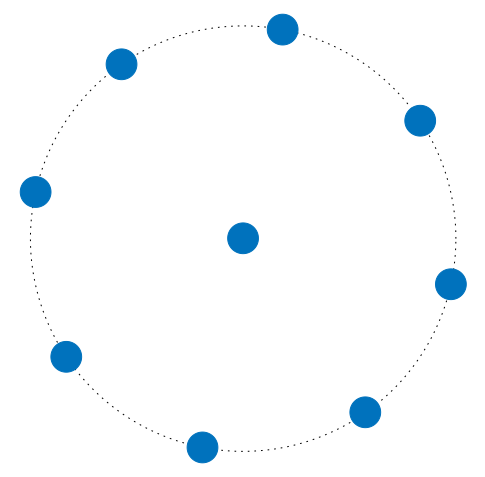

(e) sub5

Figure 9 Case study 2: optimized wind farm layouts with 9 wind turbines. 


\section{References}

[1] Herbert-Acero, J. F., Probst, O., Réthoré, P.-E., Larsen, G. C., and Castillo-Villar, K. K., "A Review of Methodological Approaches for the Design and Optimization of Wind Farms," Energies, 2014, p. 23.

[2] Sanderse, B., “Aerodynamics of Wind Turbine Wakes," Energy Resource Center of the Netherlands, 2009 , p. 46.

[3] Padrón, A. S., Stanley, A. P. J., Thomas, J. J., Alonso, J. J., and Ning, A., "Polynomial Chaos to Efficiently Compute the Annual Energy Production in Wind Farm Layout Optimization,” Wind Energy Science, 2018. doi:10.5194/wes-2017-56, (in review).

[4] Larsen, G. C., “A Simple Stationary Semi-Analytical Wake Model,” Tech. rep., Technical University of Denmark, 2009.

[5] Vermeer, L., Sorensen, J., and Crespo, A., "Wind Turbine Wake Aerodynamics," Progress in Aerospace Sciences, Vol. 39, 2003, pp. 467-510.

[6] Ott, S., Berg, J., and Nielsen, M., “Linearised CFD Models for Wakes,” Tech. rep., Technichal University of Denmark, 2011.

[7] Jensen, N., “A Note on Wind Generator Interactions,” Tech. rep., Risø National Laboratory, 1983.

[8] Gebraad, P. M. O., Teeuwisse, F. W., van Wingerden, J. W., Fleming, P., Ruben, S. D., Marden, J. R., and Pao, L. Y., "Wind Plant Power Optimization Through Yaw Control Using a Parametric Model for Wake Effects - a CFD simulation study," Wind Energy, 2014, p. 20.

[9] Thomas, J., Gebraad, P., and Ning, A., "Improving the FLORIS Wind Plant Model for Compatibility with Gradient-Based Optimization," Wind Engineering, Vol. 41, No. 5, 2017, pp. 313-329. doi:10.1177/0309524X17722000.

[10] Nocedal, J., and Wright, S. J., Numerical Optimization, $2^{\text {nd }}$ ed., Springer, 2006.

[11] Kramer, O., Ciaurri, D. E., and Koziel, S., Comput. Optimization, Methods and Algorithms, Springer, 2011.

[12] McWilliam, M. K., Zahle, F., and Dykes, K., "IEA Task 37 on System Engineering in Wind Energy The Aerodynamic Only Optimization Case Study," , May 2017.

[13] Bortolotti, P., Dykes, K., Merz, K., Sethuraman, L., and Zahle, F., "IEA Wind Task 37 on System Engineering in Wind Energy, WP2 - Reference Wind Turbines," Tech. rep., National Renewable Energy Laboratory (NREL), Golden, CO., May 2018.

[14] Bastankhah, M., and Porté-Agel, F., "Experimental and Theoretical Study of Wind Turbine Wakes in Yawed Conditions," J. Fluid Mech., Vol. 806, 2016, pp. 506-541.

[15] Thomas, J. J., and Ning, A., "A Method for Reducing Multi-Modality in the Wind Farm Layout Optimization Problem,” Journal of Physics: Conference Series, Vol. 1037, The Science of Making Torque from Wind, Milano, Italy, 2018, p. 10.

[16] Niayifar, A., and Porté-Agel, F., “Analytical Modeling of Wind Farms: A New Approach for Power Prediction,” Energies, 2016.

[17] Gill, P., Murray, W., and Saudners, M., "SNOPT: an SQP Algorithm for Large-Scale Constrained Optimization,” SIAM Review, Vol. 47, 2005, pp. 99-131.

[18] "Worth of kWh per state,", November 2018. URL https://www. chooseenergy.com/electricity-rates-by-state/. 